\title{
Normal transmission of phonons with anomalous disper- sion through the interface of two continuous media
}

\author{
I.N. Adamenko ${ }^{1,2}$, K.E. Nemchenko ${ }^{1}$, and I.V. Tanatarov ${ }^{3}$ \\ ${ }^{1}$ V.N. Karazin Kharkov National University, 4 Svobody Sq., Kharkov 61077, Ukraine \\ ${ }^{2}$ Electro-Physical Scientific and Technical Centre of the National Academy of Sciences of Ukraine \\ 28 Chernyshevsky Str., Kharkov 61002, Ukraine \\ ${ }^{3}$ Akhiezer Institute for Theoretical Physics National Science Center \\ «Kharkov Institute of Physics and Technology» of the National Academy of Sciences of Ukraine \\ 1 Academicheskaya Str., Kharkov 61108, Ukraine \\ E-mail: igor_tanatarov@inbox.ru
}

Received August 4, 2005

\begin{abstract}
In this work the problem is solved of normal transmission of quasiparticles through the interface of two continuous media, one of which is quantum fluid. The quantum fluid is described as a continuous medium with correlations. Within the framework of this approach the dispersion relation of the quantum fluid $\Omega(k)$ can be arbitrary. The integral equation describing it in a half-space is solved by the Wiener-Hopf method, and its general solution is obtained. This approach is applied to the dispersion relation of the Bose-Einstein condensate. It is shown that the solutions of equations of quantum fluid in a half-space are traveling waves deformed near the border by specific surface standing waves. By means of boundary conditions the general solution in the whole space is obtained. Expressions for transmission and reflection factors of waves in both directions are derived, depending on their frequency. The results are important for describing the creation of helium II phonons on the boundary with a solid, and are of interest for classical acoustics.
\end{abstract}

PACS: 67.40.Bz, 67.40.Db, 67.40.Pm

Keywords: superfluidity, quasiparticles, dispersion, interface

\section{Introduction}

Many physical properties of continuous media at low temperatures can be described in terms of quasiparticles which are observed experimentally. So, for example, in the experiments of $[1,2]$ the quasiparticles of superfluid helium were created on the interface with a heated solid. Then the propagation of quasiparticles in superfluid helium and their reflection from various interfaces was investigated.

When incident on an interface of two continuous media, a quasiparticle brings to it its energy and is destroyed. On both sides of the interface new quasiparticles are created with certain probabilities, and carry away from the interface the energy of the destroyed one. We shall name the described process briefly «transfer of quasiparticles through the interface». This process underlies a series of observable phenomena. In particular, it determines the temperature discontinuity (Kapitza's discontinuity, see, for example, [3]) which always exists on the boundary of two continuous media at low temperatures.

The dispersion relation of real continuous media $\Omega(k)$ is nonlinear. Direct experiments [1,2] have shown, that in superfluid helium there are phonons with anomalous dispersion, which is close to linear, and rotons with dispersion essentially different from linear. The dispersion relation of quasiparticles of Bose-Einstein condensate (BEC), according to [4], at small wave vectors $k$ is close to linear, and with growth of $k$ becomes strongly nonlinear. Meanwhile the problem of quasiparticles' transfer through the interface of two continuous media has been solved till now only for the case of strictly linear dispersion laws of both the adjacent media. 
The aim of the present work is the rigorous solution of this problem for the case when the dispersion relation of one of the media is nonlinear. Thus the description of Kapitza's temperature discontinuity [3] is generalized to the case of nonlinear dependence $\Omega(k)$. In this work we consider the dispersion relation of BEC - the quantum continuous medium, the experimental realization of which has led to occurrence of numerous experimental and theoretical works devoted to the research of its physical properties (see, for example, the reviews $[5,6])$. The dispersion relation of $\operatorname{BEC} \Omega(k)$ at small wave vectors $k$ well describes the dispersion relation of the phonons of superfluid helium. That is why the results obtained in this work may appear important not only for BEC, but also for research on mechanisms of creation and evolution of phonon beams in superfluid helium. In recent years a number of experimental and theoretical works devoted to these questions have been published (see, for example, [7-10]).

The results obtained appear to be important also for classical acoustics, where the problem of wave passage through the interface has been solved only for the case when the dispersion relations of both the adjacent media are strictly linear (see, for example, [11]). This problem with respect to real media, that have nonlinear dispersion, was of interest in the middle of the last century (see, for example, [12]) and is still relevant. For example, in recent works [13,14] simulations of wave packets transfer through the interfaces of real crystals were carried out. In [13] the passage of phonon wave packets through the interface of two semiconductors was investigated by means of molecular dynamics simulations. In [14] the phonons transport in superlattices made up of the same crystals was investigated.

We describe a quantum fluid with nonlinear dispersion relation within the framework of the theory suggested in [15], in which it is considered as a continuous medium with correlations. This theory is based on the fact that the thermal de Broglie wavelength of a particle of a quantum fluid exceeds the average interatomic separation. Then the variables of the continuous medium can be assigned values at each mathematical point of space in the probabilitic sense, but the relations between them become nonlocal. This nonlocality allows us to describe a continuous medium with arbitrary dispersion relation. The approach was also used earlier in [16] to describe the hybridization of rotons and ripplons of superfluid helium, but without a substantiation for the possibility of application of this theory to the description of a quantum fluid.

It turned out that the theory introduced in [15], which describes a quantum fluid in the infinite space, cannot be trivially generalized to the half-space. In the brief work [17] a way of such generalization was offered, and the equation describing a quantum fluid in half-space was obtained. One of the solutions of this equation was obtained there (here we name it the «out-solution») that corresponds to wave packets traveling from the interface. In this work the two linearly independent solutions of the equation (the inand out-solutions) are obtained. This enabled us to solve the problem of the phonon passage not only from a solid into the quantum liquid, but also in the opposite direction. Besides, only the in-solution gives us the phase shifts of waves at their reflection from the interface of a quantum fluid with vacuum or with an infinitely hard solid. We find that the probabilities of quasiparticles passage through the solid-quantum fluid interface in both directions are equal at the same frequencies. This means that at equal temperatures of the continuous media on both sides of the interface there exists the thermodynamic equilibrium between them - the heat flows to the right and to the left are compensated.

In section 2 of this work we, following [17], formulate the problem; we derive the equation describing a quantum fluid in a half-space and introduce the solution of this equation for arbitrary dispersion relation of the quantum fluid obtained by Wiener-Hopf method [18]. In theoretical physics this method was used earlier, in particular, for the description of guided electromagnetic waves [19]. It has been applied to the solution of the equations of quantum fluid in half-space for the first time. This solution is of interest by itself and is given in detail in the mathematical appendix.

In section 3, working from the general solution, we investigate the dispersion relation $\Omega(k)$ of Bose-Einstein condensate. At small wave vectors this dispersion law well describes the anomalous dispersion relation of phonons of superfluid helium. It is shown that if the dispersion relation of the continuous medium differs from linear, then specific standing waves appear near the boundary which correspond to the imaginary roots of equation $\Omega^{2}(k)=\omega^{2}$. Neither these surface waves nor the traveling waves which correspond to the real roots of equation $\Omega^{2}(k)=\omega^{2}$ are by themselves the solutions of the equations of a continuous medium with correlations in the half-space. The solutions of the equations of quantum fluid in the halfspace are certain linear combinations of them traveling waves that are distorted near the interface by additives that decay exponentially deep into the medium. The parameters of the solution that are determined by the properties of the continuous medium receive physical interpretation in terms of the microscopic theory of BEC in subsection 3.2. 
In subsection 3.3 we obtain the solution in the whole space with the help of boundary conditions and derive the amplitude reflection and transmission factors of wave packets passing through the interface, depending on their frequency. Using them, we obtain the probabilities of creation of quasiparticles on the interface. From them we get the correction to the creation probability of any quasiparticles with anomalous dispersion relation at small wave vectors. In particular, this gives us the correction to the transmission factor through the solid-superfluid helium interface at small $k$.

In subsection 3.4 we take into account that far from the boundary the energy in a quantum fluid is transported in a wave packet with the group velocity [20] and show that in our solution the energy is conserved. This is not a trivial fact because the standard, and it could seem simpler methods (see, for example, [16]), appear to lead to such solutions in which energy is not conserved.

\section{Formulation of the problem, derivation of equations, and their solution for arbitrary disper- sion relation}

Let us consider two continuous media separated with a sharp interface $x=0$. To the left of the interface, in the region $x<0$, there is an ordinary continuous medium with linear dispersion relation $\Omega_{\text {sol }}(k)$. Its equilibrium density is $\rho_{\text {sol }}$, sound velocity is $s_{\text {sol }}$. The one-dimensional problem is solved, in which the variables of continuous medium $\rho_{s}, V_{s}$, and $P_{s}$ depend on one coordinate $x$ and time $t$. Here $\rho_{s}$ is the deviation of density from the equilibrium value, $V_{s}$ is the velocity of continuous medium and $P_{s}$ is the deviation of pressure from the equilibrium value. They are related to each other through the equations of continuous medium (see system (2)), in which the relation between pressure and density is local

$$
P_{s}(x, t)=s_{\text {sol }}^{2} \rho_{s}(x, t) .
$$

The solution of these equations is well known and gives the linear dependence of frequency of the characteristic oscillations on their wave vector $\Omega(k)$

$$
\Omega_{\text {sol }}^{2}(k)=s_{\text {sol }}^{2} k^{2} .
$$

The region $x>0$, to the right of the boundary, is filled with a quantum fluid with a nonlinear dispersion relation $\Omega(k)$ that can be essentially nonlinear. We describe it with the help of the nonlocal hydrodynamics developed in [15]. According to it, in the infinite space the quantum fluid obeys the ordinary linearized equations of a continuous medium:

$$
\left\{\begin{array}{l}
\dot{\rho}=-\rho_{0} V^{\prime} \\
\dot{V}=-\frac{1}{\rho_{0}} P^{\prime}
\end{array}\right.
$$

where $\rho_{0}$ is the equilibrium density of the quantum fluid, in which density depends on pressure through a nonlocal relation

$$
\rho(x)=\int_{-\infty}^{\infty} d \xi h(\xi-x) P(\xi) .
$$

Here the dot indicates a derivative with respect to time $t$, and the prime a derivative with respect to $x$. Fourier transform of system (2) and (3) gives us a simple relation between the Fourier transform of the kernel of the nonlocal relation $h(x)$

$$
H(k)=\int_{-\infty}^{+\infty} d x \mathrm{e}^{-i k x} h(x)
$$

and the dispersion relation of the medium [15] $\Omega(k)$ :

$$
H(k)=\frac{k^{2}}{\Omega^{2}(k)} .
$$

The suggested model describes well the interface between a quantum fluid (superfluid helium or BEC) and a solid, because on the frequency scale of the dispersion curve of helium II (and all the more so for BEC) the dispersion curves of most solids, such as $\mathrm{Cu}$ or $\mathrm{Au}$, are very close to linear and these solids can be described as ordinary continuous media. In the problem considered, that of normal incidence on the interface, the transverse waves in a solid can be neglected.

Equations (2) and (3) give an equation for pressure

$$
P^{\prime \prime}(x, t)=\int_{-\infty}^{\infty} d \xi h(\xi-x) \ddot{P}(\xi, t), \quad x \in(-\infty, \infty),
$$

which is the generalization of the wave equation to the case of a nonlinear dispersion relation and describes a continuous medium with correlations in the infinite space.

To formulate the problem for the case when such a medium fills a confined region, we consider the interface sharp, so that the kernel of nonlocal relation (3) stays the same in the presence of the surface. Then if we take into account the fact that the fluid fills the region $x \in(0, \infty)$, we get the problem stated on the half-line:

$$
P^{\prime \prime}(x, t)=\int_{0}^{\infty} d \xi h(\xi-x) \ddot{P}(\xi, t), \quad x \in(0, \infty) .
$$


This is an integro-differential equation with difference kernel, given on the half-line $x \in(0, \infty)$, not on the whole real line. In this form it does not contain a convolution product in the sense of the Fourier transform because of the finite lower limit. There is no convolution product in the sense of either the Laplace or one-sided Fourier transform because the function $h(x)$ is even [15] and cannot become zero at $x<0$. In order to solve equation (7) by the Fourier transform we have first to define the unknown function in the region $x<0$ and to widen the limits of integration to infinity.

However, here we cannot just search for an even (or odd) solution, as is often done when solving differential equations. The wave equation describing an ordinary continuous medium in the infinite space as well as in a confined geometry has symmetry with respect to the substitution $x \rightarrow-x$. This gives us reason to search for a solution with the same symmetry. But the integral equation that describes continuous medium with correlations in a half-space (7) does not have such symmetry. Therefore we cannot claim the function $P(x)$ to be even, and if we make this assumption, we may encounter a contradiction. Indeed, let us demand the equation (7) to be true for $P(x)$ on the whole interval $x \in(-\infty,+\infty)$. Then the left side of Eq. (7) for any $x \in(-\infty, \infty)$ can be obtained from the right side, and the right side is defined on this interval only by the values of the function $P(x)$ at $x \in(0,+\infty)$. So the values of $P(x)$ at $x \in(-\infty, 0)$ are completely determined by the values of $P(x)$ at $x \in(0,+\infty)$. Then the function $P(x)$ on the whole interval $x \in(-\infty,+\infty)$, composed of those two functions, does not have to be either odd or even.

This means that if we follow the authors of [16] and try to find an even solution of the equation from problem (7) stated on the real line, not on the half-line as in (7), we will come to a mathematical problem different from the initial one (7), and its solution will not be the solution of problem (7). Our estimations with the even solution showed that energy is not conserved in it. Exceptions are the cases of reflection from vacuum and from an infinitely hard surface, but in those cases the solutions themselves are also different from ours. This means that the mathematical problem with the demand for an even function is not adequate to the initial physical formulation (7). Therefore we solve this equation on $x \in(0, \infty)$ with the help of the Wiener-Hopf method. This method does not use any additional assumptions, and as we shall see it gives us a unique solution in which energy is conserved.
The solution, given in the appendix, leads to the two linearly independent solutions of problem (7) with different asymptotes:

$$
\begin{aligned}
P_{\text {out }}^{-}(k, \omega) & =\frac{C_{\text {out }}(\omega)}{k-k_{1}(\omega)} \prod_{k_{i} \in \mathbf{C}_{+}}^{\prime} \frac{k-k_{i}(\omega=0)}{k-k_{i}(\omega)}, \\
P_{\text {in }}^{-}(k, \omega) & =\frac{C_{\text {in }}(\omega)}{k+k_{1}(\omega)} \prod_{k_{i} \in \mathbf{C}_{+}}^{\prime} \frac{k-k_{i}(\omega=0)}{k-k_{i}(\omega)} .
\end{aligned}
$$

Here the function $P^{-}(k, 0)$ is the Fourier transform of a new function $P^{-}(x, t)$, that is equal to $P(x, t)$ at $x>0$ and to zero at $x<0$. The products are taken over all the roots $k_{i}$ of the equation $\Omega^{2}(k)=\omega^{2}$ in the upper half-plane $\mathbf{C}_{+}$of the complex variable $k$. The real roots are assumed to be shifted from the real line in accordance to the rule (A.14) for the out-solution, and in the opposite direction for the in-solution. The primes over the products designate that the «main» root is omitted in them - it is $k_{1}(\omega)$ for the out-solution and $(-1) k_{1}(\omega)$ for the in-solution. Taking into account the roots shifting, those main roots lie in the upper half-plane for the both solutions and are obtained continuously from zero with the increase of 0 . The functions $C_{\text {in }}$ and $C_{\text {out }}$ are determined by boundary conditions.

Let us consider the extreme case of low frequencies ( $) \rightarrow 0$, when the dispersion relation is close to linear $\Omega^{2}(k) \approx s^{2} k^{2}$. In this case the products in relations (8) and (9) tend to unity, $k_{1} \approx \omega / s$, and those expressions are brought to the form

$$
P_{\text {out }}^{-}(k, \omega) \approx \frac{C_{\text {out }}(\omega)}{k-\omega / s} ; \quad P_{\text {in }}^{-}(k, \omega) \approx \frac{C_{\text {in }}(\omega)}{k+\omega / s} .
$$

Then we make the inverse Fourier transform by $x$, shifting the roots up, and obtain

$$
\begin{aligned}
& P_{\text {out }}^{\operatorname{lin}}(x, \omega) \approx i C_{\text {out }}(\omega) \exp \left(i \frac{\omega}{s} x\right) ; \\
& P_{\text {in }}^{\operatorname{lin}}(x, \omega) \approx i C_{\text {in }}(\omega) \exp \left(-i \frac{\omega}{s} x\right) .
\end{aligned}
$$

Those are the well-known solutions for the simplest special case of linear dispersion relation, that correspond to a set of plane waves traveling from the interface $\left(P_{\text {out }}\right)$ or towards it $\left(P_{\text {in }}\right)$. The expressions $(8)$ and (9) give the generalization of these solutions to the case of nonlinear dispersion relation of the continuous medium. 


\section{Transfer of wave packets through the boundary with a quantum fluid}

\subsection{In- and out-solutions for a quantum fluid on the half-line}

Bose-Einstein condensate is an example of a quantum fluid with anomalous dispersion relation. Its experimental realization has lead to a large number of experimental and theoretical works devoted to investigation of its physical properties (see, for example, the reviews $[5,6])$.

In Bogoliubov's theory [4] the dispersion relation $\Omega(k)$ of the quasiparticles of BEC in the approximation of point interaction has the form

$$
\Omega^{2}(k)=s^{2} k^{2}\left(1+\frac{k^{2}}{2 k_{0}^{2}}\right) .
$$

Here $s=\sqrt{n \alpha / m}$ is the sound velocity; $k_{0} / s=$ $=\sqrt{2} \mathrm{~m} / \hbar ; \alpha$ is the matrix element of the binary interaction of the particles of BEC; $m$ is their mass; $n$ is their number per unit volume.

The dispersion relation (12) is the simplest complication with regard to the linear case in the framework of our approach to the description of continuous media with nonlinear dispersion relation. At small $k$ this relation approximates the dispersion of any continuous medium with anomalous dispersion relation, that is when $\Omega(k)$ at small $k$ deflects upward from the linear law. This also applies to superfluid helium at a range of pressures.

The equation $\Omega^{2}(k)=\omega^{2}$ with $\Omega^{2}(k)$ from Eq. (12) has four roots: $\pm k_{R}$ and $\pm k_{I}$, where

$$
\left\{\begin{array}{l}
k_{R}(\omega)=k_{0} \sqrt{\Gamma-1}, \\
k_{I}(\omega)=i k_{0} \sqrt{\Gamma+1} .
\end{array}\right.
$$

Here the symbols are introduced

$$
\Gamma=\sqrt{1+\chi^{2}}, \text { where } \chi=\omega \sqrt{2} / k_{0} s
$$

is the dimensionless frequency.

Then the general solution (8), (9) applied to the dispersion relation (12) gives

$$
P_{\text {in }}^{-}(k, \omega)=\frac{C_{\text {in }}(\omega)}{k \pm k_{R}(\omega)} \frac{k-k_{m}}{k-k_{I}(\omega)},
$$

where $k_{m}=k_{I}(\omega=0)=i k_{0} \sqrt{2}$. The upper sign corresponds to the in-solution, the lower sign - to the out-solution. On shifting $k_{R}(\omega)$ from the real line (A.14) and taking the inverse Fourier transform by $x$, we get
$P_{\text {out }}(x, \omega)=P_{\text {out }}(0, \omega)\left\{\mathrm{e}^{i k_{R} x} \frac{k_{R}-k_{m}}{k_{R}-k_{I}}+\mathrm{e}^{i k_{I} x} \frac{k_{m}-k_{I}}{k_{R}-k_{I}}\right\}$,

$P_{\text {in }}(x, \omega)=P_{\text {in }}(0, \omega)\left\{\mathrm{e}^{-i k_{R} x} \frac{k_{R}+k_{m}}{k_{R}+k_{I}}+\mathrm{e}^{i k_{I} x} \frac{k_{I}-k_{m}}{k_{R}+k_{I}}\right\}$.

We consider the wave packets passing through the interface, and that means that the pressure on the interface oscillates with frequencies close to the carrier frequency of the wave packet $\omega_{0}$. So

$$
P_{\text {out }}(0, t)=\tilde{P}(t) \mathrm{e}^{-i \omega_{0} t} ; P_{\text {in }}(0, t)=\hat{P}(t) \mathrm{e}^{-i \omega_{0} t},
$$

where functions $\tilde{P}(t)$ и $\hat{P}(t)$ are the envelopes of packets in time, that change much more slowly with time than the oscillating parts.

Now we expand the quantities in the exponents of Eqs. (16) и (17) into series by $\left(\omega-\omega_{0}\right)$ and restrict ourselves to the linear terms, the coefficients by the exponents are taken at $\omega=\omega_{0}$. Such expansion was used in works $[21,22]$ in order to investigate the wave packets of electromagnetic field and it gives the description of a packet's propagation without deformation.

We omit the index 0 at $\omega_{0}$ and have in mind here and below that $k_{I}$ and $k_{R}$ are taken at the carrier frequency $\omega=\omega_{0}$. Then taking the inverse Fourier transform with respect to $t$ we get

$$
\begin{aligned}
& P_{\text {out }}(x, t)=\frac{k_{R}-k_{m}}{k_{R}-k_{I}} \tilde{P}\left(t-\frac{x}{u_{R}}\right) \mathrm{e}^{i k_{R} x-i \omega t}+ \\
& +\frac{k_{m}-k_{I}}{k_{R}-k_{I}} \tilde{P}(t) \mathrm{e}^{-k_{I} \mid x-i \omega t}, \\
& P_{\text {in }}(x, t)=\frac{k_{R}+k_{m}}{k_{R}+k_{I}} \hat{P}\left(t+\frac{x}{u_{R}}\right) \mathrm{e}^{-i k_{R} x-i \omega t}+ \\
& +\frac{k_{I}-k_{m}}{k_{R}+k_{I}} \hat{P}(t) \mathrm{e}^{-k_{I} \mid x-i \omega t} .
\end{aligned}
$$

Here $u_{R}=u\left(k_{R}\left(\omega_{0}\right)\right)$ is group velocity of the wave packet with carrier frequency $\omega_{0}$.

As we know from the equations of continuous media (2) the relation between the Fourier transforms of pressure and velocity

$$
V(k, \omega)=\frac{1}{\rho_{0}} \frac{k}{\omega} P(k, \omega),
$$

we can put down from solutions (19) and (20) the expressions for velocity in the in- and out-solutions: 


$$
\begin{aligned}
& V_{\text {out }}(x, t)=\frac{1}{\rho_{0}} \frac{k_{R}}{\omega} \frac{k_{R}-k_{m}}{k_{R}-k_{I}} \tilde{P}\left(t-\frac{x}{u_{R}}\right) \mathrm{e}^{i k_{R} x-i \omega t}+ \\
& +\frac{1}{\rho_{0}} \frac{k_{I}}{\omega} \frac{k_{m}-k_{I}}{k_{R}-k_{I}} \tilde{P}(t) \mathrm{e}^{-\left|k_{I}\right| x-i \omega t} \\
& V_{\text {in }}(x, t)=-\frac{1}{\rho_{0}} \frac{k_{R}}{\omega} \frac{k_{R}+k_{m}}{k_{R}+k_{I}} \hat{P}\left(t+\frac{x}{u_{R}}\right) \mathrm{e}^{-i k_{R} x-i \omega t}+ \\
& +\frac{1}{\rho_{0}} \frac{k_{I}}{\omega} \frac{k_{I}-k_{m}}{k_{R}+k_{I}} \hat{P}(t) \mathrm{e}^{-\left|k_{I}\right| x-i \omega t} .
\end{aligned}
$$

According to Eqs. (19), (20), (22) and (23), structurally the solutions consist of two parts - a traveling wave packet (toward the interface in the in-solution and away from it in the out-solution) and a standing wave that exponentially decreases with distance from the boundary. Thus the solution that in the infinite space has the form of a traveling wave, is distorted in the presence of the boundary by the additives that decrease exponentially from it. It must be emphasized that neither the traveling nor standing waves are the solutions of equation (7) by themselves.

The coefficients of all the summands in Eqs. (19), (20), (22), and (23) are essentially complex, which means that there are phase shifts between all the waves. The range of penetration $\left|k_{I}\right|^{-1}$ of the standing waves into the medium is of the order of the wavelength of the travelng part $\left|k_{R}\right|^{-1}$ and therefore is much less than the size of the wave packet. At this scale the correction $\pm x / u_{R}$ to the arguments of $\hat{P}(t)$ and $\tilde{P}(t)$ in the corresponding terms are not essential and therefore are omitted. We can see from the expression for $k_{I}$ (13) that at small frequencies the penetration depth of the standing wave is approximately constant and is of the order of $k_{0}^{-1}$, and at $\chi \rightarrow \infty$ it tends to zero as $1 / \sqrt{\chi}$. The physical interpretation of the penetration depth will be discussed in subsection 3.2.

Let us consider the contributions of standing and traveling waves to the pressure and velocity on the interface. We denote the ratio of the standing waves' contribution to $P(0, t)$ to the contribution of the traveling waves as $\pi$, and the same ratio for the contributions to $V(0, t)$ as $v$. The subscripts «in» and «out» designate those ratios for the in- and out-solutions corresponding. Then we can obtain these quantities directly from Eqs. (19), (20), (22), and (23). With the help of relations (13) we can show that $\pi_{\text {in }}=\pi_{\text {out }}^{*}$, $v_{\text {in }}=v_{\text {out }}^{*}$, where the star means complex conjugation, and

$$
\left\{\begin{array}{l}
\left|\pi_{\text {out }}\right|=\left|\pi_{\text {in }}\right|=1-\sqrt{\frac{2}{\Gamma+1}}, \\
\left|v_{\text {out }}\right|=\left|v_{\text {in }}\right|=\frac{\sqrt{\Gamma+1}-\sqrt{2}}{\sqrt{\Gamma-1}}, \\
\arg \pi_{\text {out }}=-\arg \pi_{\text {in }}=-\arcsin \sqrt{\frac{\Gamma-1}{\Gamma+1}}=-\arcsin \left|\frac{k_{R}}{k_{I}}\right|, \\
\arg v_{\text {out }}=-\arg v_{\text {in }}=\arccos \sqrt{\frac{\Gamma-1}{\Gamma+1}}=\arccos \left|\frac{k_{R}}{k_{I}}\right| .
\end{array}\right.
$$

According to Eqs. (24), at small frequencies, $\chi<<1$, the contributions of standing waves are small and $\pi_{\text {in,out }} \approx \chi^{2} / 8, v_{\text {in,out }} \approx \pm i \chi / 4$. As the frequency increases, their contributions also increase and become comparable with the contributions of the traveling waves. At large frequencies the ratios of the contributions of standing and traveling waves to velocity and pressure on the interface tend to unity as $1 / \sqrt{\chi}$ : at $\chi \gg 1$ $\pi_{\mathrm{out}} \approx-i+(1+i) \sqrt{2 / \chi}, v_{\mathrm{out}} \approx 1-(1-i) \sqrt{2 / \chi}$.

The relative phases of velocity and pressure oscillations in all the waves also depend on frequency. For the in-solution the picture on the phase diagram is the following. The velocity in the traveling wave oscillates in antiphase with pressure. The velocity in the standing wave is shifted by $(-\pi / 2)$ relative to the pressure in it. At $\chi<<1$ the phase difference between the pressures of standing and traveling waves $\arg \pi_{\text {in }}$ is small and positive and it rises monotonically with increase in frequency. At $\chi \rightarrow \infty$ the phase shift between pressures of standing and traveling waves tends to $\pi / 2$ and the velocities are in antiphase. For the out-solution the scheme differs only by the sign of the relative phase of pressures of standing and traveling waves.

\subsection{Correlation length and penetration depth}

In order to describe a quantum fluid in general, and the Bose-Einstein condensate in particular, we used a model in which nonlinearity of the dispersion relation is equivalent to nonlocality of the relation between pressure and density with kernel $h(x)$. As we know the relation (5) between the Fourier image of the kernel $H(k)$ with the dispersion relation $\Omega(k)$, we can easily obtain this kernel. For the dispersion of BEC (12) in the one-dimensional case it is

$$
h(x)=\frac{k_{m}}{2 s^{2}} \mathrm{e}^{-\left|k_{m}\right||x|} .
$$

We see that the correlation length $\lambda$ is of the order of

$$
\lambda \sim\left|k_{m}\right|^{-1} \sim k_{0}^{-1} .
$$


If we express $k_{0}$ in terms of the microscopic theory of BEC, we can express it in the form $\lambda \sim \hbar / m s$. This quantity may be interpreted as the de Broglie wavelength of a particle with mass $m$ moving with the sound velocity $s$. If we use the expression for the sound velocity in BEC, we will get $\lambda \sim \hbar / \sqrt{\alpha m n}$, which is the de Broglie wavelength of a particle of mass $m$ with energy $\varepsilon_{p} \approx \alpha n$, equal to the average energy of a particle's interaction with the condensate. The penetration depth of the standing wave into the condensate $\delta$, in accordance with Eq. (16), is equal to $\left|k_{I}(\chi)\right|$. At $\chi<<1$ it is nearly constant and of the order of $\left|k_{m}\right|$, and therefore is of the order of the correlation length $\lambda$. Thus in the limit of small frequencies there is only one quantity with the dimensions of length that characterizes the system, i.e., the correlation length $\lambda(26)$.

At large frequencies the penetration depth tends to zero as $\delta(\chi) \approx\left(k_{0} \sqrt{\chi}\right)^{-1}$. On substituting $k_{0}$ and $\chi$, we

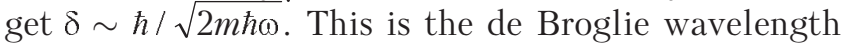
of a free particle of mass $m$ and energy $\hbar \omega$. In the limit of large frequencies this is also the de Broglie wavelength of the condensate quasiparticle of frequency $\omega$.

\subsection{Reflection and transmission factors}

We will call the amplitude reflection factor $r$ the ratio of the envelopes of pressures in the reflected and the incident wave packet. In the quantum fluid the envelopes are taken of the traveling parts of the solutions, which transport energy far from the interface. As the packets are composed of sets of plane waves with close frequencies, evidently the plane wave with the carrier frequency has the same reflection factor. We obtain the reflection and transmission factors with the help of boundary conditions that demand continuity of pressure and velocity on the interface.

Let us at first consider the problem of a wave packet incident on the interface from the solid. In this case there are both the incident and the reflected waves in the solid (region $x<0$ ), and in the quantum fluid (region $x>0$ ) there exists only the out-solution.

As the time dependence of all the solutions is the same, the boundary conditions can be satisfied only if all the frequencies are equal. Then the incident and the reflected waves have equal frequencies and wave vectors that differ only in sign. This is enough to express the reflection factor $r$ through pressure and velocity on the interface:

$$
r=\frac{Z-1}{Z+1}, \text { where } Z=\frac{P(0, t) / V(0, t)}{\rho_{\mathrm{sol}} s_{\mathrm{sol}}} .
$$

In the well-known case, when the region $x>0$ is filled with an ordinary continuous medium, with density $\rho_{0}$, a linear dispersion relation, and sound velo- city $s_{0}, Z$ turns into the real impedance of the interface $\left(\rho_{0} s_{0}\right) /\left(\rho_{\text {sol }} s_{\text {sol }}\right)$ and then the expression for the reflection factor takes the ordinary real form.

In the general case the quantities $P(0, t)$ and $V(0, t)$ can also be found from the out-solution (19) and (22) with the help of boundary conditions. As a result the relation (27) for $Z$ gives

$$
Z=\frac{Z_{R}}{1+i \sigma},
$$

where the notations are introduced for real quantities

$$
Z_{R}=\frac{\rho_{0} s_{R}}{\rho_{\mathrm{sol}} s_{\mathrm{sol}}} \text { and } \sigma=\frac{\left|k_{I}-k_{m}\right|}{k_{R}} .
$$

According to definitions (29), $Z_{R}$ is the impedance of a boundary between a solid and an ordinary continuous medium with density $\rho_{0}$ and linear dispersion law with sound velocity $s_{R}=\omega / k_{R}$, which is the phase velocity of a wave packet with carrier frequency $\omega_{0}$. On substitution of expressions for $k_{R, I, m}$ into Eq. (29), one can see that $\sigma$ depends only on the dimensionless frequency $\chi$.

After substituting relation (28) into Eq. (27), we'll get the amplitude reflection factor for a wave incident on the interface with a quantum fluid from the solid $r_{\rightarrow}$ :

$$
r_{\rightarrow}=\frac{Z_{R}(\omega)-1-i \sigma(\chi)}{Z_{R}(\omega)+1+i \sigma(\chi)} .
$$

This quantity is complex and therefore there is a nontrivial (i.e., different from zero or $\pm \pi$ ) phase shift between the incident and reflected waves, equal to $\arg r_{\rightarrow}$.

The transmission factor $D$ is the share of the incident wave's (wave packet's) energy that is transferred through the interface into the second medium. As the share of the reflected energy is $|r|^{2}$, the transmission factor equals $D=1-|r|^{2}$. Then from Eq. (30) we obtain

$$
D=\frac{4 Z_{R}}{\left(Z_{R}+1\right)^{2}+\sigma^{2}} .
$$

This quantity can be expressed in terms of only two parameters,

$$
D\left(Z_{0}, \Gamma\right)=\frac{4 Z_{0} \sqrt{\frac{\Gamma+1}{2}}}{\left(Z_{0} \sqrt{\frac{\Gamma+1}{2}}+1\right)^{2}+\left(\frac{\sqrt{\Gamma+1}-\sqrt{2}}{\sqrt{\Gamma-1}}\right)^{2}},
$$

where 


$$
Z_{0}=\frac{\rho_{0} s}{\rho_{\mathrm{sol}} s_{\mathrm{sol}}}
$$

is the (ordinary) impedance of the boundary in the limit $\omega \rightarrow 0$ and does not depend on frequency, and $\Gamma$ is $\Gamma(\chi)$ from definition (14).

At $\omega \rightarrow 0$ (which is equivalent to $\Gamma \rightarrow 1$ ), the expression (32) turns into the classic expression for energy transmission factor through an interface between two continuous media with impedance $Z_{0}$

$$
D_{0}=\frac{4 Z_{0}}{\left(Z_{0}+1\right)^{2}} .
$$

At small frequencies $\chi<<1$ the nonlinearity brings in a small correction, quadratic in frequency:

$$
D(\chi) \approx D_{0}\left(1-\frac{\chi^{2}}{16} \frac{2 Z_{0}^{2}-1}{\left(Z_{0}+1\right)^{2}}\right) .
$$

This expression gives the correction for the transmission factor through the interface with any continuous medium, that has an anomalous dispersion relation (i.e., it deflects upward from the linear law) at small frequencies $\chi<<1$.

At $\chi \gg 1$ the factor $D$ slowly tends to zero

$$
D(\chi) \approx \frac{4}{Z_{0}} \sqrt{2 / \chi}
$$

There are many interfaces, for example between solids and liquids or solids and gases, for which the strong inequality $Z_{0} \ll 1$ is true. The transmission factor through such interfaces with liquids that have anomalous dispersion relation at small frequencies is given by Eq. (35), in which we should put $Z_{0}=0$.

In particular, the dispersion relation of superfluid helium at small frequencies has the form

$$
\Omega(k) \approx s k\left(1+\gamma k^{2}\right) .
$$

Therefore for the transmission factor with superfluid helium at small $k$ we obtain

$$
D(k) \approx D_{0}\left(1+\frac{1}{2} \gamma k^{2}\right) .
$$

Now let us consider the problem of a wave packet incident on the interface from the quantum fluid. The solution in the quantum fluid (region $x>0$ ) then is a sum of in- and out-solutions

$$
P_{\leftarrow}(x, t)=P_{\text {in }}(x, t)+P_{\text {out }}(x, t) \text { at } x>0,
$$

and in the solid (region $x<0$ ) there is only the transmitted wave.

As we know the relation between velocity and pressure in a plane wave (21), which is the only wave in the solid in this case, we can take into account the sign of its wave vector and obtain that $P(0, t) / V(0, t)=$ $=-\rho_{\mathrm{sol}} S_{\mathrm{sol}}$. From the other side of the boundary we find $P(0, t)$ from Eq. (39), on substituting there $P_{\text {out }}$ and $P_{\text {in }}$ from Eqs. (19) and (20). Similarly, we get $V(0, t)$ taking $V_{\text {out }}$ and $V_{\text {in }}$ from Eqs. (22) and (23). Then the quantity $P(0, t) / V(0, t)$ is expressed in terms of the ratio $\tilde{P}(t) / \hat{P}(t)$, and we find the last one using the boundary conditions. The reflection factor is found as the ratio of the coefficients at $\exp \left( \pm i k_{R} x\right)$ in solutions (19) and (20):

$$
r_{\leftarrow}(\omega)=\frac{1-Z_{R}+i \sigma}{1+Z_{R}-i \sigma} \cdot \frac{k_{R}-k_{m}}{k_{R}+k_{m}} \cdot \frac{k_{R}+k_{I}}{k_{R}-k_{I}} .
$$

If we use Eqs. (29), (33) and (13), the expression (40) can be reduced to

$$
\begin{aligned}
& r_{\leftarrow}\left(Z_{0}, \Gamma\right)=\frac{1-Z_{0} \sqrt{\frac{\Gamma+1}{2}}+i \frac{\sqrt{\Gamma+1}-\sqrt{2}}{\sqrt{\Gamma-1}}}{1+Z_{0} \sqrt{\frac{\Gamma+1}{2}}-i \frac{\sqrt{\Gamma+1}-\sqrt{2}}{\sqrt{\Gamma-1}}} \times \\
& \times \frac{\sqrt{\Gamma-1}-i \sqrt{2}}{\sqrt{\Gamma-1}+i \sqrt{2}} \cdot \frac{\sqrt{\Gamma-1}+i \sqrt{\Gamma+1}}{\sqrt{\Gamma-1}-i \sqrt{\Gamma+1}} .
\end{aligned}
$$

According to Eq. (41), there is a phase shift $\arg r_{\leftarrow}$ between the incident and the reflected waves when a packet falls on the interface from the quantum fluid, and it is different from arg $r_{\rightarrow}$.

It is important that the absolute values of reflection factors in both directions coincide $\left|r_{\rightarrow}\right|=\left|r_{\leftarrow}\right|$. Then the energy transmission factors in both directions are the same and equal to $D$ from Eq. (31). This leads to the fact that at equal temperatures on both sides of the interface the heat flows in opposite directions are compensated and the continuous media are in thermodynamic equilibrium.

Let us now consider the extreme cases of reflection of quasiparticles of a quantum fluid from the free surface and the opposite extreme case of its reflection from an infinitely hard surface. The reflection factor at the reflection from vacuum is obtained from Eq. (41) by passing to the limit $Z_{0} \rightarrow \infty$. This is true if the phonon's energy is small enough not to cause quantum evaporation at the free surface [2]. Reflection from an infinitely hard wall is also obtained from Eq. (41) if we put $Z_{0}=0$. We can see that in both cases the absolute value of the reflection factor goes to 1 (and therefore the transmission factor to zero), but its argument remains nontrivial. So, in both cases there are standing waves by the surface of the quantum fluid, and phase shifts between the incident and the reflected waves. 


\subsection{Energy flows. Conservation laws}

Working from the general solutions in the two adjacent continuous media, with the help of boundary conditions we obtain the solution in all the interval $x \in(-\infty,+\infty)$. As there are two boundary conditions, we can express all the amplitudes in terms of one of them, for example, the amplitude of the incident wave.

The expression for the energy flow in an ordinary liquid is commonly known. The expression for the energy flow density in a wave packet of a quantum fluid in the infinite space $Q_{\infty}(x, t)$ was obtained in the work [20]. It was shown in [20], that in a quantum fluid with nonlinear dispersion relation the energy of a wave packet is transported with the group velocity, so that

$$
\left\langle Q_{\infty}(x, t)\right\rangle=u_{R}\left\langle\varepsilon_{\infty}(x, t)\right\rangle .
$$

Here $\varepsilon_{\infty}(x, t)$ is the energy density in the wave packet. It was defined in [15] as $\varepsilon_{\infty}=\rho_{0} V^{2} / 2+$ $+\rho P / 2 \rho_{0}$. The brackets in Eq. (42) indicate averaging over rapid oscillations. The expression (42) was derived in the way similar to the one used in [21] and [22], where the propagation of wave packets and transport of energy in them was investigated for an electromagnetic field in a medium. Thus we can express the energy flows in the incident, reflected and transferred wave packets in terms of one amplitude. But then the conservation of energy is not trivial and requires verification.

It can be shown from the general solution in the ordinary continuous medium that the average energy flow through the interface when a wave packet is passing through it in any direction is equal to

$$
\left\langle Q_{S}(t)\right\rangle=\langle P(0, t) V(0, t)\rangle .
$$

Let us consider the problem of a wave packet incident on the interface from the solid. Then we can find $P(0, t)$ and $V(0, t)$ from the out-solutions in the quantum fluid (19) and (22) if we put $x=0$. When we substitute those expressions into Eq. (43), we get

$$
\left\langle Q_{s}(t)\right\rangle=\frac{|\tilde{P}(t)|^{2}}{2 \rho_{0} s_{R}} .
$$

From the other side, far enough from the boundary, at $x \gg\left|k_{I}\right|^{-1}$, where the influence of interface can be neglected, the average energy flow density $\left\langle Q_{\infty}\right\rangle$ is defined by Eq. (42). Then it can be brought to the same form as in an ordinary continuous medium $\rho_{0}\left\langle V^{2}(x, t)\right\rangle$. When we substitute here the envelope of the velocity in the traveling wave packet from Eq. (22), use the expressions for $k_{R}, k_{I}$ and $u_{R}$, and compare the result with Eq. (44), we find that

$$
\left\langle Q_{\infty}(x, t)\right\rangle=\left\langle Q_{s}\left(t-x / u_{R}\right)\right\rangle .
$$

This equality means that the energy brought to the interface by the incident wave packet is taken away by the reflected and the transmitted packets, i.e., that energy is conserved in the process. It can be shown in a similar manner that energy is conserved when a wave packet passes through the interface in the opposite direction as well. This result is not at all trivial, as it is easy to check that if we search for an even solution (as in [16], for example), the energy in the general case will not be conserved.

\section{Conclusions}

In this work we have solved the problem of normal transmission of quasiparticles through the interface of two media, one of which is a quantum fluid. It is solved as a problem of wave packets transmission through a boundary of an ordinary continuous medium and a continuous medium with correlations [15]. In the framework of this approach the continuous medium with correlations may have arbitrary dispersion relation $\Omega(k)$. The solution in the whole space is obtained with the help of boundary conditions that demand pressure and velocity continuity on the interface. The equations of quantum fluid in half-space are brought to an integro-differential equation (7), which is solved by the Wiener-Hopf method. Its general solution (8), (9) is obtained. In the extreme case of linear dispersion relation of the quantum fluid and in the limit of small frequencies, when dispersion law is close to linear, the solution turns into plane waves (11) and gives the well-known reflection and transmission factors for the case of linear dispersion relation (34). The general solution is investigated for the dispersion relation of quasiparticles of Bose-Einstein condensate (BEC) (12). At small frequencies it approximates well to the dispersion relation of phonons of superfluid helium (37) and of quasiparticles of other continuous media with anomalous dispersion.

It is shown that in a medium with dispersion relation $\Omega(k)$ that differs from the linear law, there always exist specific surface waves that correspond to imaginary roots of the equation $\Omega^{2}(k)=\omega^{2}$. These waves, however, are fundamentally different from the Rayleigh surface waves because they appear at normal incidence of traveling waves on the interface. They differ also from thermal waves as they are not by themselves the solutions of equations of the continuous medium with correlations. Their solutions are only definite linear combinations of ordinary traveling waves and the standing surface waves (19), (20). Thus, the solutions of equations of continuous medium with correlations are plane waves that are distorted over distances of the order of the wavelength from the boundary by exponential terms. At small frequencies 
the penetration depth of the standing waves is of the order of the correlation length (26) and it is the only quantity with dimensions of length that describes the quantum fluid. At large frequencies the penetration depth is of the order of de Broglie wavelength of a quasiparticle of BEC. There are phase shifts between the standing and traveling waves that depend on their frequency.

The amplitude reflection factors are obtained for the wave packets incident on the interface both from the solid (30) and from the quantum fluid (40). They depend on the carrier frequency of the wave packet as well as on the properties of the adjacent media. The complexity of the reflection factors leads to the existence of nontrivial (not equal to zero or $\pi$ ) phase shifts between incident and reflected waves. The extreme cases of reflection from vacuum or from infinitely hard surface are obtained from the general expression (41) if we pass to the limits $Z_{0} \rightarrow \infty$ or $Z_{0}=0$, respectively. The energy transmission factors in both directions are derived (31) and they coincide. This ensures compensation of the heat flows in both directions when temperatures on both sides of the interface are equal. Those transmission factors are the probabilities of new quasiparticles' creation on the interface and they characterize the heat exchange between a solid and a quantum fluid. A correction is obtained for the transmission factor through an interface between a solid and a medium with anomalous dispersion relation for small wave vectors (35). For the interface with superfluid helium this correction takes the form (38).

It is shown, that due to the existence of standing waves near the boundary the energy conservation, shown by Eq. (45), follows from the boundary conditions. This is not a commonplace fact because standard methods of solution of the stated problem, that seem to be also simpler, lead to solutions in which energy is not conserved (see, for example, [16]). Such contradictions appear, in particular, if we try to find an even solution of equation (7), that is stated on a half-line and does not possess that symmetry. In this work we do not make any additional assumptions of this kind and use the Wiener-Hopf method to solve the equation (7) on the half-line.

The results obtained in this work may be applied to describe Kapitza's temperature discontinuity and heat exchange between solids and various quantum continuous media (superfluid helium, BEC and others). They are necessary for understanding the mechanism of creation of phonon beams in superfluid helium by a heated solid [7-10]. The results are important for classical acoustics as well, as an example of solution of the problem of waves transmission through the interface of two continuous media in the case when dispersion relation of one of them is nonlinear and may be essentially nonlinear. Further development of this theory may give description of the transfer of quasiparticles with arbitrary dispersion relation through the interface at all incidence angles.

\section{Mathematical appendix: solution of our problem with the Wiener-Hopf method}

\subsection{Reduction of our equation to a Riemann boundary problem}

Taking the Fourier transform of Eq. (7) by $t$, we get

$$
P^{\prime \prime}(x)=-\omega^{2} \int_{0}^{\infty} d \xi h(\xi-x) P(\xi) .
$$

This is an integro-differential equation with one variable. We will use the Wiener-Hopf method to solve it. It should be noted, that the function $P(x)$ is yet defined only on $x \in(0, \infty)$. So in order to take the Fourier transform of Eq. (A.1), let us introduce two new functions $P^{-}(x)$ and $P^{+}(x)$. The first one is defined as

$$
P^{-}(x)=P(x) \text { at } x>0,
$$

and

$$
P^{-}(x)=0 \text { at } x<0 .
$$

Let us likewise demand for the second function

$$
P^{+}(x)=0 \text { at } x>0,
$$

and at $x<0$ let it be defined so that the equation

$$
\left(P^{-}+P^{+}\right)^{\prime \prime}=-\omega^{2} \int_{-\infty}^{\infty} d \xi h(\xi-x) P^{-}(\xi)
$$

should hold true on $x \in(-\infty, 0)$. At $x \in(0, \infty)$ the equation (A.4) is fulfilled automatically.

We take the Fourier transform of Eq. (A.4) by $x$

$$
P^{ \pm}(k)=\int_{-\infty}^{\infty} d x \exp \{-i k x\} P^{ \pm}(x) .
$$

We are looking for the solutions $P^{ \pm}(x)$, that grow not faster than power law at infinity. Then the definitions of $P^{ \pm}(k)$ (A.5) and demands (A.2b) and (A.3) lead to important analytical properties of these functions. Let us indicate as $\mathbf{C}_{+}$the upper half-plane of complex variable $k$ and as $\mathbf{C}_{-}$the lower half-plane. Then $P^{+}(k)$ is analytical in $\mathbf{C}_{+}$, and $P^{-}(k)$ is analytical in $\mathbf{C}_{-}$. 
Then on taking Fourier transform (A.5) of Eq. (A.4) and using the convolution theorem, we get

$$
-k^{2}\left(P^{-}(k)+P^{+}(k)\right)=-\omega^{2}\left\{H(k) P^{-}(k)\right\} .
$$

Here $H(k)$ is expressed through the dispersion relation of the quantum fluid $\Omega(k)$ through relation (5) [15]. Then relation (A.6) is brought to the form

$$
P^{-}(k) \frac{\Omega^{2}(k)-\omega^{2}}{\Omega^{2}(k)}+P^{+}(k)=0 .
$$

This equality defines on the real line $k \in(-\infty,+\infty)$ a linear relation between the limit values of functions $P^{+}$and $P^{-}$on it, that are analytical correspondingly in the upper and lower half-planes of the complex variable $k$. Therefore it defines a Riemann boundary problem for the half-plane.

\subsection{Solution of Riemann boundary problem without singularities on the contour}

In its simplest form the Riemann boundary problem is formulated as follows (see, for example, [18]).

There is a simple smooth closed contour $L$ that divides the complex plane $z$ into the interior domain $D^{+}$ and the exterior domain $D^{-}$. $G(p)$ (the Riemann problem factor) is a function of the points of the contour which satisfies the condition of Goelder and doesn't go to zero. We need to find the two functions: $\Phi^{+}(z)$, analytical in the domain $D^{+}$, and $\Phi^{-}(z)$, analytical in domain $D^{-}$including $z=\infty$, that satisfy on the contour $L$ the linear relation

$$
\Phi^{+}(p)=G(p) \Phi^{-}(p) .
$$

A function $G(p)$ is said to satisfy the Goelder condition on contour $L$, if for any $p_{1}, p_{2} \in L$ there are such $A>0$ and $\lambda \in(0,1]$, that

$$
\left|G\left(p_{1}\right)-G\left(P_{2}\right)\right|<A\left|p_{1}-p_{2}\right|^{\lambda} \text {. }
$$

Index of the function $G(p)$ is the increment of its argument at path-tracing the contour, divided by $2 \pi$. It can be expressed through the logarithm

$$
\text { Ind } G(p)=\frac{1}{2 \pi}[\arg G(p)]_{L}=\frac{1}{2 \pi i} \int_{L} d \ln G(p) \text {. }
$$

If $G(p)$ satisfies the listed conditions and remains real on the contour, then evidently Ind $G(p)=0$. In this case it can be shown [18], that it follows from relation (A.8) that the functions $\Phi^{+}(z)$ and $\Phi^{-}(z)$ do not turn into zero in the domains of their analyticity. Then on taking the logarithm of Eq. (A.8), we get

$$
\ln \Phi^{+}(p)-\ln \Phi^{-}(p)=\ln G(p),
$$

where $\ln G(p)$ satisfies the Goelder condition, $\ln \Phi^{+}(p)$ and $\ln \Phi^{-}(p)$ are single-valued on $L$, and $\ln \Phi^{+}(z)$ and $\ln \Phi^{-}(z)$ are analytical correspondingly in $D^{+}$and $D^{-}$.

Then Eq. (A.11) represents the problem of finding a piecewise analytical function from its step [18]. Its solution is given by the formula of Sohotskij [18] and it is defined up to an additive constant:

$$
\ln \Phi(z)=\frac{1}{2 \pi i} \int_{L} \frac{\ln G(p)}{p-z} d p+\text { const } .
$$

Here $\ln \Phi(z)$ is a piecewise analytical function, equal to $\ln \Phi^{+}(z)$ at $z \in D^{+}$and to $\ln \Phi^{-}(z)$ at $z \in D^{-}$.

This solution can be extended [18] to the case when contour $L$ is the real line, $D^{+}=\mathbf{C}_{+}$, and $D^{-}=\mathbf{C}_{-}$.

\subsection{Complications in our problem with regards to the simplest case. Roots shifting}

The factor of the Riemann boundary problem (A.7) is

$$
J(k)=\frac{\Omega^{2}(k)-\omega^{2}}{\Omega^{2}(k)} .
$$

It turns into zero in the roots of equation $\Omega^{2}(k)=\omega^{2}$. Also we are interested in the dispersion relation such that $\Omega^{2}(k) \sim k^{2}$ at $k \rightarrow 0$, so at this point the factor $J(k)$ is non-bounded and therefore does not satisfy the Goelder condition. It can be seen from (A.13), that at $k \rightarrow \infty$ function $J(k)$ is bounded and separated from zero. So the problem (7) contains two complications with regard to problem (A.8). First, the factor $J(k)$ turns into zero in the points $k$ of the real line, such that $\Omega^{2}(k)=\omega^{2}$. Second, function $J(k)$ is unbounded at $k=0$.

The first complication is eliminated this way: we assume that the points $k$, in which $\Omega^{2}(k)=\omega^{2}$, always have at least a small imaginary part, so that $J(k)$ turns into zero not on the contour.

The sign of this addition is found from the demand of damping of any waves in the direction of their propagation. If we search for a solution that should contain wave packets running from the interface, the rule for shifting of a root $k_{j} \in(-\infty,+\infty)$ from the real line can be put down symbolically in the form

$$
k_{j}=k_{j}+i \operatorname{sign} u_{j} \cdot 0,
$$

where $u_{j}=\left.(d \Omega(k) / d k)\right|_{k_{j}}$ is the group velocity in the point $k=k_{j}$. This means that the points $k_{j}$ are shifted in accordance with the signs of group velocities in them. If $u_{j}>0$, the point is shifted up, if $u_{j}<0-$ down. We will call such solutions the «out-solutions».

In a similar way we may search for solutions that far from the interface represent wave packets running 
to the interface. Those will be «in-solutions» and for them the rule for roots shifting will be the opposite. If $u_{j}>0$, the root $k_{j}$ is shifted down, and if $u_{j}<0$ it is shifted up. As we take $\Omega(-k)=\Omega(k)$, in both cases there are as many roots shifted up as down. So Ind $J(k)$ remains equal to zero.

\subsection{Solving Riemann boundary problem with singularities on the real line}

Let us now eliminate the second complication, associated with the unboundedness of factor $J(k)$ at zero. We define a new function $I(k)$ such that

$$
J(k)=\frac{A^{2}+k^{2}}{k^{2}} I(k),
$$

where $A>0$ is an arbitrary number. It can be seen from (A.15) that Ind $I(k)=$ Ind $J(k)=0$.

As $I(k)$ satisfies, after the roots shifting, all the conditions laid on $G(k)$ in problem (A.8), the Riemann boundary problem (A.8) on the real line with the factor $I(k)$ instead of $J(k)$ can be solved by the scheme given in subsection 5.2. In other words, $I(k)$ can be in a unique way (up to a multiplicative constant) presented in the form

$$
I(k)=\frac{I^{-}(k)}{I^{+}(k)},
$$

where $I^{+}(k)$ is analytical in $\mathbf{C}_{+}, I^{-}(k)$ is analytical in $\mathbf{C}_{-}$and in accordance with subsection 5.2 they do not turn into zero in the domains of their analyticity.

Until now the solution did not require any restriction on the form of the dispersion relation of our continuous medium with correlations $\Omega(k)$. But the asymptotical behavior of a real relation $\Omega(k)$ at $k \rightarrow \infty$ must not have any influence on the solution of the problem of transmission of a wave packet with a finite carrier frequency though a boundary. From the other side, any real dispersion relation can be approximated on a finite interval of $k$ by a polynomial function of arbitrary power with any required precision.

Then, without reducing the generality of our consideration, we may assume $\Omega^{2}\left(k^{2}\right)$ to be a polynomial. In this case the procedure of factorization of $I(k)$ (A.16) is significantly simplified with regard to the general procedure given in subsection 5.2. The difference $\left(\Omega^{2}(k)-\omega^{2}\right)$ can be presented in the form

$$
\Omega^{2}(k)-\omega^{2}=B \prod_{i}\left(k^{2}-k_{i}^{2}(\omega)\right),
$$

where the product is taken over all the roots $k_{i}^{2}$ of equation $\Omega^{2}(k)=\omega^{2}$ relative to $k^{2}$ in the complex plane.
As we consider such dispersion relation, that $\Omega^{2}(k) \sim k^{2}$ at $k \rightarrow 0$, on taking into account Eq. (A.17), $\Omega^{2}(k)$ can be presented in the form

$$
\Omega^{2}(k)=B k^{2} \prod_{i}^{\prime}\left(k^{2}-k_{i}^{2}(\omega=0)\right) .
$$

Here the prime over the product means that it does not contain the factor with $k_{j}^{2}(\omega=0)=0$, which is taken out of the product. When we substitute Eqs. (A.17) and (A.18) into relation (A.13) and take into account definition (A15), we get

$$
I(k)=\frac{I^{-}(k)}{I^{+}(k)}=\frac{1}{k^{2}+A^{2}} \frac{\prod_{i}\left(k^{2}-k_{i}^{2}(\omega)\right)}{\prod_{i}^{\prime}\left(k^{2}-k_{i}^{2}(\omega=0)\right)} .
$$

Every term of the products $k^{2}-k_{i}^{2}=\left(k-k_{i}\right) \times$ $\times\left(k+k_{i}\right)$ contains one multiplier with its zero in $\mathbf{C}_{+}$ and one with zero in $\mathbf{C}_{-}$. If we group together all the factors of the numerator and the denominator that turn into zero in $\mathbf{C}_{+}$, we'll get a function that is analytical in $\mathbf{C}_{-}$and doesn't go to zero in this domain. But, taking into account subsection 5.2, those are the properties of function $I^{-}(k)$, which is defined only within a multiplicative constant. Therefore

$$
I^{-}(k)=\frac{1}{k-i A} \frac{\prod_{\mathbf{C}_{+}}\left(k-k_{i}(\omega)\right)}{\prod_{\mathbf{C}_{+}}^{\prime}\left(k-k_{i}(\omega=0)\right)},
$$

where the product is taken over all the roots of equation $\Omega^{2}(k)=\omega^{2}$ with regard to $k$ in $\mathbf{C}_{+}$. In the product with prime the factor $\left(k-k_{j}(\omega=0)\right)$ is omitted with $k_{j} \in \mathbf{C}_{+}$such that $k_{j}(\omega) \rightarrow+0$ at $\omega \rightarrow 0$. In the in- and out-solutions those factors are different. Indeed, let us indicate as $k_{1}(\omega)$ that real positive root of $\Omega^{2}(k)=\omega^{2}$ (if it exists), in which the root $k_{j}(\omega=0)=0$ passes continuously when $\omega$ increases from zero. Then, as $\Omega(k)=\Omega(-k), u\left(k_{1}\right)>0$ and $u\left(-k_{1}\right)<0$. In accordance to our rule for roots shifting (A.14), for the out-solution the root $k_{1}$ is shifted up and $(-1) k_{1}$ down; for the in-solution $(-1) k_{1}$ is shifted up and $k_{1}$ is shifted down.

Then if we factor out the corresponding multiplier from the product of the numerator, we get finally for $I^{-}$in the in- and out-solutions (here the upper signs correspond to the in-solutions, and the lower signs to the out-solutions)

$$
I_{\text {in }}^{-}(k)=\frac{k \pm k_{1}(\omega)}{k-i A} \prod_{k_{i} \in \mathbf{C}_{+}} \frac{k-k_{i}(\omega)}{k-k_{i}(\omega=0)} .
$$


Taking into account Eqs. (A.15) and (A.16), we can rewrite the equality (A.7), given on $k \in(-\infty, \infty)$, in the form

$$
(k-i A) P^{-}(k) I^{-}(k)=-\frac{k^{2}}{k+i A} P^{+}(k) I^{+}(k) .
$$

The left side of equality (A.22) is the limit value on $k \in(-\infty, \infty)$ of a function analytical in $\mathbf{C}_{-}$, and the right side is the limit value of a function analytical in $\mathbf{C}_{+}$. Then by the analytic continuation theorem those functions are analytical continuations of each other over the real line and are equal to some function $\Psi(k)$, analytical in the whole complex plane, with exception possibly only in the points on the real line and complex infinity. It is fully determined by those poles.

We demand for the solution to be fully determined by the properties of the continuous medium and the physical statement of the problem. Then we rely on the fact that the dispersion relation of a medium $\Omega(k)$ defines completely its dynamical properties and postulate that the equality (A.22) contains all the physical factors determining the poles of $\Psi(k)$. So we search for the functions $P^{ \pm}(k)$, that may possibly have poles in the complex plane only in the poles and zero points of $J(k)$ (its zero points are the roots of $\Omega^{2}(k)=\omega^{2}$, and the only pole is of the second order in zero) and of no greater order. Then $\Psi(k)$ must be analytical on $k \in(-\infty, \infty)$ and can have poles only at zero or infinity.

At $x \rightarrow+\infty$ the solution must turn into the wellknown solution in the infinite medium [15], which is the set of plane waves. The behavior of $P(x)$ at $x \rightarrow+\infty$ is determined by the asymptote of $P^{-}(k)$ at $k \rightarrow 0$ and then it can be seen from Eq. (A.22), that $\Psi(k)$ must be bounded at zero.

For the problem stated for the function $P(x)$ to be fully determined by equation (7), we should look for $P(x)$ in the category of functions, that do not contain $\delta$-function or its derivatives at zero. In the opposite case we should specify how to understand the lower limit of integration in Eq. (7), which is quite baffling. Then we should demand $P^{-}(k)=O(1 / k)$ at $k \rightarrow \infty$, and so $\Psi(k)$ must be bounded at infinity. All the foregoing means eventually that we are searching for the solution $P^{ \pm}(k)$, bounded on the contour, as it is usually done when solving mathematical problems of this kind [18].

Then $\Psi(k)$ is analytical in the whole complex plane, including infinity, and by the (generalized) Liouville theorem (see, for example, [18]) is equal to a constant:

$$
\begin{aligned}
& (k-i A) P^{-}(k) I^{-}(k)=-\frac{k^{2}}{k+i A} P^{+}(k) I^{+}(k)= \\
& =\Psi(k)=C(\omega) .
\end{aligned}
$$

The last equality is true for all $k \in \mathbf{C}$. It gives us the solution of the boundary problem (A.7):

$$
P^{-}(k)=\frac{C(\omega)}{I^{-}(k)(k-i A)} .
$$

The constant $A$ is only formally present in Eq. (A.24), as the factor $(k-i A)$ on factorization (A.20) always gets into the denominator of $I^{-}(k)$.

When we substitute $I^{-}$from Eq. (A.21) into Eq. (A.24), we finally get the expressions for the Fourier transforms of pressures in the in- and out-solutions (9) and (8).

\section{Acknowledgements}

We would like to thank A.F.G. Wyatt for many helpful discussions. We also express our gratitude to EPSRC of the UK (grant EP / C 523199/1), and to GFFI of Ukraine (grant N02.07/000372) for support for this work.

1. A.F.G. Wyatt, N.A. Lockberie, and R.A. Sherlock, Phys. Rev. Lett. 33, 1425 (1974).

2. M.A.H. Tucker and A.F.G. Wyatt, Science 283, 1150 (1999).

3. I.M. Khalatnikov, An Introduction to the Theory of Superfluidity Addison-Wesley, New York (1998).

4. N.N. Bogoliubov, J. Phys. USSR 11, 23 (1947); reprinted in D. Pines, The Many Body Problem Benjamin, New York (1961).

5. C.J. Pethick and H. Smith, Bose-Einstein Condensation in Dilute Gases, Cambridge University Press, Cambridge (2002).

6. L. Pitaevskii and S. Stringari, Bose-Einstein Condensation, Clarendon Press, Oxford (2003).

7. Ruslan V. Vovk, Charles D.H. Williams, and Adrian F.G. Wyatt, Phys. Rev. B68, 134508 (2003).

8. Ruslan V. Vovk, Charles D.H. Williams, and Adrian F.G. Wyatt, Phys. Rev. Lett. 91, 235302 (2003).

9. I.N. Adamenko, K.E. Nemchenko, V.A. Slipko, and A.F.G. Wyatt, Fiz. Nizk. Temp. 30, 579 (2004) [Low Temp. Phys. 30, 432 (2004)].

10. I.N. Adamenko, K.E. Nemchenko, V.A. Slipko, and A.F.G. Wyatt, J. Phys.: Condens. Matter 17, 2859 (2005).

11. L.M. Brehovskih, Waves in Layer Media, Nauka, Moskow (1973).

12. L.I. Mandelshtamm, Lectures on Optics, Relativity Theory and Quantum Mechanics, Nauka, Moskow (1972).

13. P.K. Schelling, S.R. Phillpot, and P. Keblinski, Appl. Phys. Lett. 80, 2484 (2002). 
14. P.K. Schelling and S.R. Phillpot, J. Appl. Phys. 93, 5377 (2003)

15. I.N. Adamenko, K.E. Nemchenko, and I.V. Tanatarov, Phys. Rev. B67, 104513 (2003).

16. L. Pitaevskii and S. Stringari, Phys. Rev. B45, 13133 (1992).

17. I.N. Adamenko, K.E. Nemchenko, and I.V. Tanatarov, J. Low Temp. Phys. 138, 10909-005-1584-9 (2005).

18. F.D. Gahov, Boundary Problems, Gos. izdat. fiz-mat. lit., Moskow (1958).
19. R. Mittra and S.W. Lee, Analytical Techniques in the Theory of Guided Waves, The MacMillan Company, New York (1971).

20. I.N. Adamenko, K.E. Nemchenko, and I.V. Tanatarov, J. Mol. Liquids 120, iss. 1-3, p. 167 (2005).

21. S.M. Rytov, Zh. Eksp. Teor. Fiz. 17, 930 (1947).

22. Ya.L. Alpert, Izvestia Akademii Nauk SSSR, seria. Fiz. 12, 241 (1948). 